\title{
Kulturgeschichte und Dermatologie
}

\section{Cultural History and Dermatology}

\section{Bibliografie}

DOI http://dx.doi.org/

10.1055/s-0029-1243899

Online-Publikation: 22. 2. 2010

Akt Dermatol 2010; 36:

192-195 @ Georg Thieme

Verlag KG Stuttgart · New York ISSN 0340-2541

Korrespondenzadresse

Prof. Ernst G. Jung

Maulbeerweg 20

69120 Heidelberg

ernst.g.jung@t-online.de

\section{Zusammenfassung \\ $\nabla$}

Die Haut und damit die Dermatologie haben ihren Ursprung in der Kulturgeschichte, und zwar in der frühen Zeit der Entstehung der Mythen. Dies kann am Mythos des geschundenen Satyrs Marsyas beispielhaft gezeigt werden. Er ist bis heute wirkkräftig. Der Leichnam erfüllt die Metapher für Dominanz, Unterdrückung und für den Ver-

\section{Einleitung}

\section{$\nabla$}

Die Wertschätzung der Haut und die kulturhistorische Bedeutung derselben, die Verankerung also in der Erinnerung, geht zurück in die Frühzeit der Menschheitsgeschichte. Es ist dies diejenige frühe Phase, in welcher auch die Mythen entstanden sind. Die Haut erscheint als ein starkes Element in den Mythen in vielfältiger Art und mannigfachem Bezug. Es lohnt sich, diesen nachzugehen. Es lassen sich vielfältige Wirkungen auf die gegenwärtigen Reflexionen über unsere Haut sowie deren Bedeutung und Bezüge zur modernen Dermatologie und Kosmetologie verfolgen. Dazu betrachten wir zunächst die Frühzeit mit dem Ursprung der Mythen, um anschließend die Reflexionen der Moderne zu beleuchten, einschließlich des Phänomens der „Gegenwartsschrumpfung“.

\section{Frühzeit}

\section{$\nabla$}

Der heutige moderne Mensch (Homo sapiens) hat vor 170000 Jahren Afrika in Richtung Norden verlassen (out of Africa). Nach der letzten Eiszeit, im Holozän also, breitet er sich über die ganze Erde aus. Als Nomade, Sammler und Jäger, sowie als

* Nach dem Festvortrag an der Jahrestagung der AG Psychosomatische Dermatologie der DDG am 27. November 2009 in Berlin-Spandau. lust von Person und Individualität. Die leere Hülle der Haut allerdings steht für Fruchtbarkeit und rhythmische Erneuerung nach dem Symbol der sich häutenden Schlange. Solches wirkt bis heute weiter in der Psychodermatologie, der neuen Philosophie der Haut und in der aufkommenden Debatte über die Qualitäten des Lebens, wie Schönheit, Hässlichkeit und Alterung.

kühner Seefahrer erobert er alle Kontinente. Als Siedler sodann domestizieren unsere Vorfahren Haustiere, betreiben Ackerbau und Handwerk. Es beginnt die neolithische Revolution ( $\bullet$ Tab. 1). In den Stammesgemeinschaften und der Verdichtung der Siedlungen entsteht „Kultur“. Das Leben des Menschen wird hinterfragt, die zeitlichen Abfolgen und deren Rhythmen. Die Umwelt wird als Gabe erfahren, Schicksal und Menschenwerk werden Begriffe. Mythen entstehen zur Bewältigung der schwer fassbaren Vielfalt und religiöse Elemente formieren sich. Diese zeigen zuweilen verblüffend ähnliche Inhalte und Stufen der Abfolge in räumlich getrennten Kristallisationsfeldern, den fruchtbaren Flussebenen (Zweistromland, Nil, Indus etc.).

Unsere Vorfahren überstanden die enormen klimatischen Herausforderungen, bewältigten geologische Katastrophen wie die Abtrennung Englands vom Kontinent und die Flutungen der Ostsee und des Schwarzen Meeres und sie verankerten das Geschehen in der kulturellen Erinnerung. Das menschliche Werden, Wachsen, Vermehren, das Altern und der Tod werden erlebt, beobachtet und eingeordnet als Elemente eigener Weltbilder. Die biologischen Regelmäßigkeiten werden erkannt, hinterfragt und als Gaben oder Strafen der Gottheiten gewertet. Abweichungen (Missbildungen und Krankheiten), Lebensbrüche und auch der Tod werden erkannt und göttlicher Ungnade oder Strafe zugeordnet. Diesen in sakralen Hand- 
Tab. 1 Der Homo sapiens erobert die Welt, die zweite Welle „out of Africa“ und die neolithische Revolution.

\begin{tabular}{|cll|}
$\begin{array}{l}\text { Jahre vor } \\
\text { Chr. Geburt } \\
170000\end{array}$ & $\begin{array}{l}\text { Nomaden, Jäger und } \\
\text { Sammler }\end{array}$ & \\
\hline 12000 & Siedler & Ende letzte Eiszeit \\
\hline 9000 & Neolithische Revolution & Haustiere, Getreide \\
\hline 7500 & Jericho & erste Stadt \\
\hline 6000 & Kupfer, Keramik & $\begin{array}{l}\text { Flutung Schwarzes Meer } \\
\text { Flutung Ostsee }\end{array}$ \\
\hline 5000 & Bewässerung & Brit. Insel abgetrennt \\
\hline 4000 & Bronze, Pflug, Schrift & \\
\hline
\end{tabular}

lungen zu begegnen, wird als Sache des Priesters verstanden. Krankheiten, vor allem selbstheilende und solche mit zeitlicher Begrenzung, sind neben der Begleitung Sterbender von besonderer Bedeutung für eingeweihte Heiler. Ist der Krankheitsablauf erkannt, sind die Heilungsriten so festzulegen, dass sie zur richtigen Zeit Wirkung zeigen. Dies gilt als Beweis für die Allmacht der Gottheit und auch für die spezielle Beziehung des Heilers zu dieser.

Medizin entstand in sozialen Gemeinschaften schon sehr früh durch erfüllte und effektive Heilbehandlungen im sakralen Kontext. Vom „Sacerdos“ (Opferbringer) trennt sich der Heiler (Medizinmann) durch Spezialisierung ab. Abkunft und Nähe zum Sakralen bleiben dem Mediziner allerdings noch lange erhalten. Haut allerdings gibt es schon immer, die gepflegt und geschmückt werden will. Haut spielt zudem eine gewichtige und nachhaltige psychosoziale Rolle, seit jeher und noch immer. Die Bedeutung und Gewichtung der Haut stammt aus der mythischen Zeit, wird in den Mythen tradiert und mythologisch gewertet schon in der Frühzeit und immer wieder durch die Jahrtausende bis heute, immer aktuell und immer bezogen auf den Träger der Haut, den Menschen also.

Haut ist Teil moderner Anthropologie: nämlich Decke, Dekor und Deutung.

Die Haut ist Decke; sie gibt Form, Aspekt, bildet Grenze, ist Organ der Empfindung, des Schmerzens und bei Verlust zerläuft die Persönlichkeit gleichsam. Der Mythos vom geschundenen Satyr Marsyas wird eine zentrale Figur.

Dies sei kurz erinnert: Die Göttin Athena schuf die Doppelflöte, den Aulos, aus Knochen vom Steinbock und spielte ihn ergreifend. Dennoch verwarf sie das Instrument, als sie im Wasser sah, wie sie beim Blasen entstellt wurde. Wer immer die Flöte aufhebe, sollte schwer bestraft werden. Der Satyr Marsyas hob die Flöte trotz Warnung auf und spielte sie meisterlich. Ehrgeizig fordert er den Leier spielenden Gott Apollo zum Wettstreit - und verliert! Der Sieger dürfe mit dem Besiegten nach Belieben verfahren. Auf Hautabziehen hatte man sich geeinigt. Also hängte Apollo den klagenden Marsyas an einen Baum und zog ihm bei lebendigem Leib die ganze Haut ab (so weit Ovid [1]).

Das Häuten oder Schinden war und blieb über lange Zeit eine qualvolle Strafe mit Todesfolge und Verlust der Person ( $\bullet$ Abb. 1). Zudem ist es eine Warnung vor Überheblichkeit gegenüber Göttern und Herrschern.

Marsyas gilt als Metapher in Richtung Unterdrückung und Beherrschung des Menschen durch allmächtige Dominanz. Marsyas ist Symbol für die Aufgabe der eigenen Gestalt und damit der Individualität, der Person. Der Mythos des Marsyas findet sich in allen anthropologischen Reflexionen über Dominanz auf der einen und Selbstaufgabe auf der anderen Seite.

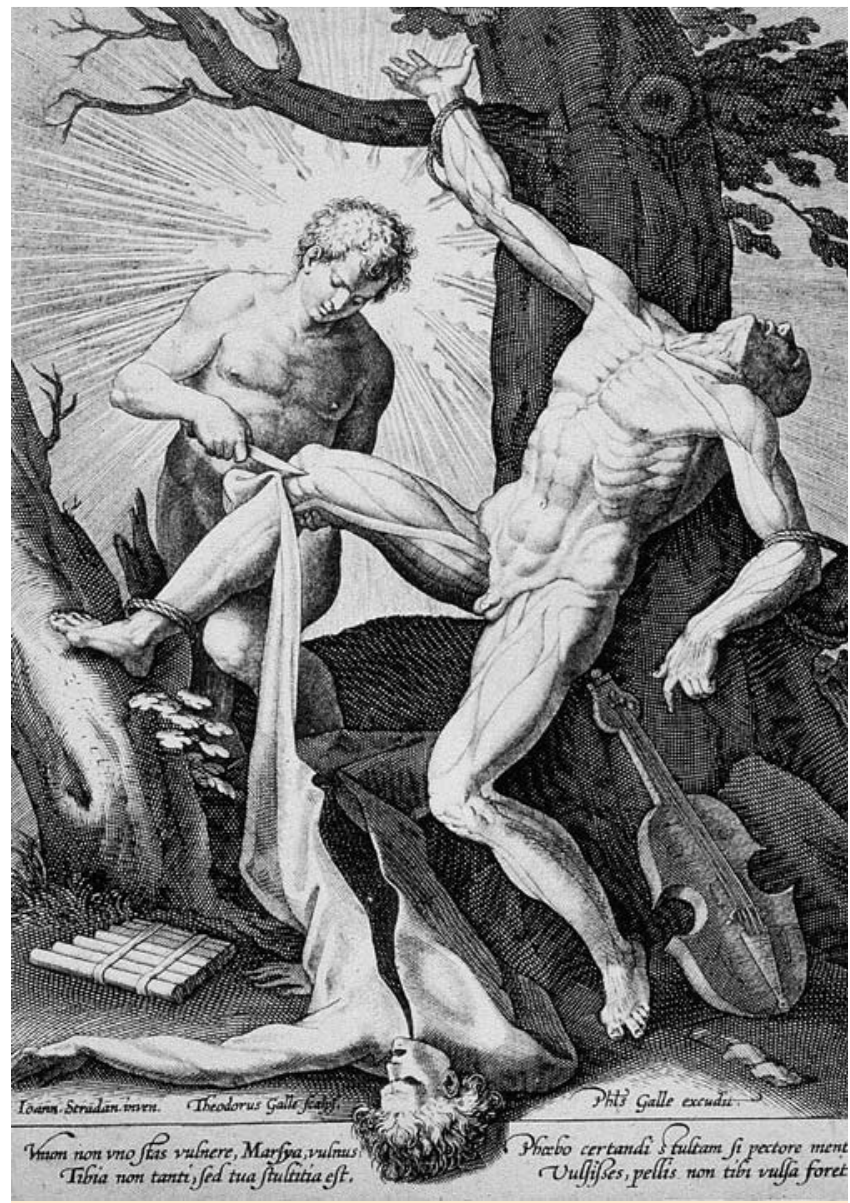

Abb. 1 Der Gott Apollo schindet den Satyr Marsyas. Während der zu Tode geschundene Marsyas am Baum gebunden verendet und seine Identität (symbolisiert durch das Wegdrehen des Kopfes) verliert, bleibt die abgetrennte Haut insgesamt erhalten und gewinnt eigenen Symbolcharakter der rhythmischen Erneuerung. Kupferstich von Theodor Gelle um 1580.

Das Schinden des Marsyas und anderer Märtyrer zieht sich durch die Jahrhunderte künstlerischer Darstellung ( $\bullet$ Abb. 2 und 3). Soviel zur Haut als Decke und deren Verlust.

Seit den frühesten Zeugnissen wird die Haut als Dekor verwendet und durch flüchtige sowie permanente Zeichen, Farben und Zusätze bereichert. Schmuck und Individualität, Einmaligkeit sind tragende Motive. Dazu kommt oft auch der Wunsch, die Zugehörigkeit zu oder auch den Ausschluss aus einer besonderen Gruppierung oder Geisteshaltung darzustellen. Solche Bemühungen haben nie nachgelassen und blühen gegenwärtig wieder mächtig auf.

\section{Deutung}

$\nabla$

Die Haut dient dem Körper als Decke, bedingt die Form, den Aspekt und gewährt Individualität. Durch Schmuck und Zusätze werden diese Effekte verstärkt oder verändert.

Die enorme Bedeutung der Haut stellt sich dar beim Verlust derselben. Solches ist wegen des Besatzes an Empfindungen besonders schmerzhaft und, wenn auch protrahiert, tödlich. Schinden ist eine qualvolle Todesstrafe. Großflächige Verbrennungen auch. Damit aber nicht genug. Es geht auch die Körperform, der Aspekt und die Individualität verloren, Elemente, die bei allen Überlegungen zu einem vorstellbaren Leben nach dem Tod, Auferstehung oder Wandlung, unabdingbar sind. Schinden zerstört also 


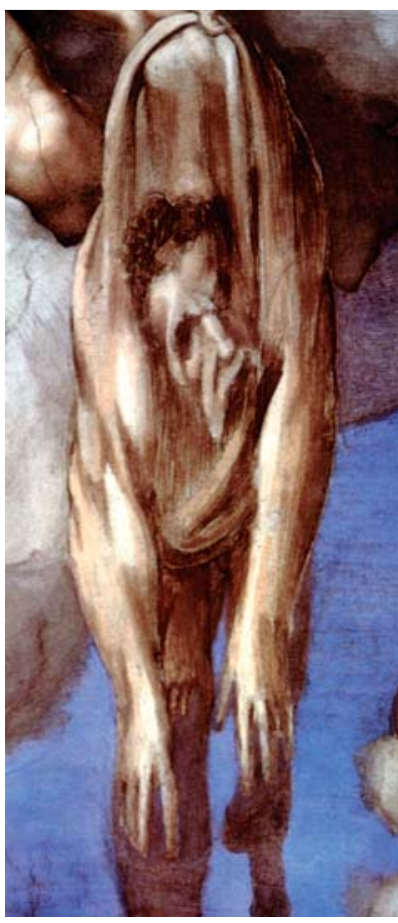

Abb. 2 Die abgetrennte Haut des Heiligen St. Bartholomäus als Symbol dauerhafter Glaubenstreue. Detail aus dem Fresco „Das jüngste Gericht“ in der Sixtinischen Kapelle von Michelangelo 1536 - 1541.

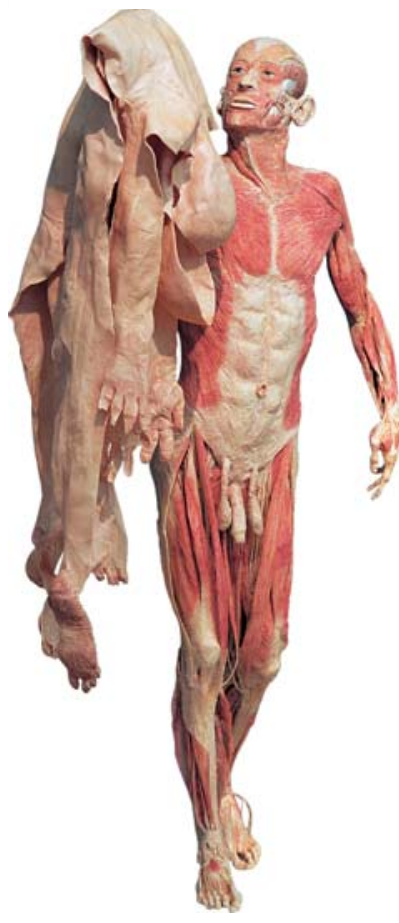

Abb. 3 Skinman, Ganzkörperplastinat von G. van Hagens, aus Körperwelten 1999. Selbstbewusst verabschiedet sich der Gehäutete von seiner Haut, die zwei Arme des Marsyas-Mythos andeutend.

zudem die erinnerbare Person und verbietet jede spirituelle Erweckung. Die Gegenwart ist der Tod, Vergangenheit wird gelöscht und Zukunft verwehrt. Auch wenn sich die Praktiken zumeist geändert haben, die Tatsachen bleiben und die Bedeutung auch. Dafür steht der Mythos vom Satyr Marsyas.

So hat Didier Anzieu 1985 (deutsch 1991) die mythische Erinnerung in typische Bausteine gegliedert, die er Schlüsselelemente nennt, und in Analogie zur Biologie als Mytheme bezeichnet [2]. Und einige von diesen Mythemen finden sich im Mythos von Marsyas. Recht zentral also!

In Tab. 2 sind solche Denk- und Ordnungsmuster skizziert und in Analogie zur Biologie gegliedert.
Es sind dies gesammelte theoretische Gedankenexperimente, welche nach analytischer Dekonstruktion komplexer Inhalte trachtet und eine endliche Zahl Bausteine als Elemente derselben postuliert. Es ist eine Zusammenführung von Hypothesen und bedarf der Überprüfung, Verwerfung oder des Beweises. Den Erfolgen in Biologie und exakten Naturwissenschaften ist es zuzuschreiben, dass die Geisteswissenschaften Ähnliches versuchen. Didier Anzieu [2] beruft sich auf den Strukturalismus von Claude Lévi-Strauss (1909-2009) und bezieht sich auf Richard Dawkins (geb. 1941), der in seinem weltbekannten Buch „The Selfish Gene“ [3] 1976 den Begriff des Mem in Analogie zum Gen einführt und typische Elmente des Denkens und der Erinnerung beschreibt (Memory-Gene sozusagen). Unschwer sind Anspielungen auf das „kulturelle Gedächtnis“ des Zeitgenossen Jan Assmann [4] zu erkennen und solche zu den Archetypen von Carl Gustav Jung (1876-1961), sowie dessen Gliederung des Unbewussten in einen persönlichen Part und einen kollektiven.

Unsere kulturelle Basis besteht offenbar und unter anderem aus zusammensetzbaren mythischen Elementen. Sie sind, wie die biologischen und psychologischen Elemente, Gene und Meme also, wohl vererbbar als auch durch mündliche oder schriftliche Übermittlung sowie durch Imitation tradierbar.

Die Verallgemeinerung basiert nicht zuletzt auf der Tatsache, dass sich ähnliche Mythen schon aus der Frühzeit vieler Kulturen nachweisen lassen und dass diese nachhaltig wirksam bleiben, bis heute.

\section{Neuzeit}

Wir verlassen die Frühzeit und ihre Ausstrahlungen und durcheilen die folgenden Jahrtausende. Die Medizin behauptet sich und bleibt spirituell, manchmal recht bizarr.

Empirie gedeiht und Spiritualität bleibt. Priester und Ärzte wirken getrennt und doch verschränkt. Wir wenden uns der Neuzeit zu und beobachten während der letzten hundert Jahre das, was die Philosophen „Gegenwartsschrumpfung“ nennen. Alles geht rasant, hektischer, mächtiger und jedes Geschehen wird verdichtet; es schrumpft die erlebte Zeit. Drei Felder sind vordringlich zu betrachten:

Die Psychodermatologie, eine Philosophie der Haut und die neue Debatte über Lebensqualitäten.

Die Medizin ist seit Rudolf Virchow naturwissenschaftlich, und sie spezialisiert sich. Dermatologie entsteht - und blüht! Die moderne Psychologie startet mit Wilhelm Wundt (1832-1920) und Sigmund Freud (1856-1939). Zusammen, Dermatologie und Psychologie, bemühen wir uns um Psychodermatologie seit gut 50 Jahren. Die „Haut als Spiegel der Seele“ gilt es zu ergründen. Haut und Hirn also mit phylogenetischem Bezug auf den gemeinsamen ektodermalen Ursprung.

Beispielhaft haben wir erfahren, dass wir Menschen

- ausgesprochen geeignete Instrumente und Fähigkeiten haben, Stresssituationen zu bewältigen, biopositiv zu gestalten und „Schulungseffekte“ auch anderen Lebensbereichen zugute kommen zu lassen. Erfahrung durch stete Forderung!

- Schwierigkeiten haben, Störungen gewohnter Rhythmen zu bewältigen und biopositiv zu nutzen. Keine kulturelle Erfahrung, Rhythmen waren stabil!

Gesichert ist zudem: Die Phase allgemeiner Zusammenhänge, Bezüge und Ausschlüsse ist überwunden. Ohne klare und differenzierte Diagnostik sowohl der Hautkrankheiten einerseits und der Psychosen anderseits geht es nicht mehr [5]. Das ist sicher. 


\begin{tabular}{|c|c|c|c|c|}
\hline Mathematik & Physik & Biologie & Psychologie & Soziologie \\
\hline Axiome & Atome & Gene & Meme & Mytheme \\
\hline Rechen-Operationen & Moleküle & Proteine & Verhaltens-Muster & Mythen \\
\hline Räume \& Zeit & Chemie & $\begin{array}{l}\text { Struktur \& } \\
\text { biol. Funktion }\end{array}$ & $\begin{array}{l}\text { Bewusstsein } \\
\text { Individualität } \\
\text { individuelles }\end{array}$ & $\begin{array}{l}\text { Gruppen-Verhalten } \\
\text { kollektives }\end{array}$ \\
\hline & & & \multicolumn{2}{|c|}{$\begin{array}{l}\text { Unbewusstsein } \\
\text { kulturelles Gedächtnis }\end{array}$} \\
\hline
\end{tabular}

Neuerdings wird mutig von der „Philosophie der Haut“ gesprochen. Spiegel der Seele und Verbindung zur Welt sind Stichworte und die Zugänge sind die hermeneutischen Erkundungen landläufiger Volksweisheiten und Sprichwörter sowie wiederum die Mythologie.

Was alles steckt dahinter, wenn wir dünnhäutig oder mit dickem Fell Fühlung aufnehmen, wenn uns Emotionen auf den Leib geschrieben, Zuneigungen unter die Haut gehen, wenn wir Schindluder treiben oder uns mit Haut und Haaren verpflichten. So zeigt sich der anthropologische Erfahrungsschatz und öffnet ganze Felder der hermeneutischen Wortdeutung [6].

Aus dem Pantheon der aztekischen Götterwelt sticht der Fruchtbarkeitsgott Xipe Totec (der sich häutet, unser Herr) hervor, der als Mensch durch einen Priester dargestellt wird, überzogen mit der Haut eines Menschenopfers. Den Häuten der dem Gott Geopferten wird Heilkraft und Erneuerung zugesprochen. Der Gott symbolisiert nach dem Bild der sich häutenden Schlange auch Wiedergeburt, Fruchtbarkeit und Überfluss [7,8]. Der Marsyas Mythos wird umgedeutet und ergänzt. Zweigestaltig wird er nun. Auf der einen Seite steht der geschundene Marsyas, ein sterbender Leichnam, als Metapher für Beherrschung und Unterdrückung des Menschen durch die allmächtige Dominanz der Herrschenden. Dazu kommt der Verlust der eigenen Person, dargestellt durch die Abwendung des Kopfes ohne einsehbares Gesicht in Abb. 1. Auf der anderen Seite ist im selben Bild die abgetrennte Haut dargestellt, bereit, eine eigene und komplementäre Figur zu bilden. Hier eine Metapher für Reifung, Verjüngung und Metamorphose nach dem Bild der sich häutenden Schlange. Durch die Häutung wird die Person getötet und gleichzeitig ausgelöscht, während die abgetrennte Haut für Fortbestand in wiederkehrenden Zyklen steht. Eine Konzentration mythischer Figuren zur wirkkräftigen Metapher für „Werden und Vergehen“.

Dies greifen Gian Condrau und Heinrich Schipperges 1993 [9] auf, wenn sie eine Philosophie der Haut als eine Anthropologie der Oberfläche, der Grenzfläche und deren Bearbeitung deuten (Schmuck, Dekor). Dies wird durch Claudia Benthien 1999 [10] und Hannelore Mittag 2001 [11] unterstützt, die kulturgeschichtlich argumentieren und zudem auf Körperbilder und Grenzen abheben. Nach wie vor ist Marsyas gegenwärtig!

Die Qualitäten des Lebens sind nicht mehr allein vorgegeben, Gott ergeben sozusagen. Nein, sie können den Vorstellungen angepasst werden. Und damit ändern sich auch die Vorstellungen. Dieser Prozess wird in typischer Weise enorm vorangetrieben, beschleunigt also, und trägt alle Zeichen der Gegenwartsschrumpfung! Wiederum aber steht die Haut im Mittelpunkt, denn sie ist beteiligt an der Körperform, gibt Aspekt und gewährt Individualität. Dekor, Kosmetik und Zutaten, können invasiv und konservativ sehr vieles anbieten, um den Ansprüchen kurzfristig nur oder permanent zu genügen. Atemraubend weiten sich die Möglichkeiten aus. Daraus ergeben sich wiederum und neu erschaffene Probleme. An beidem, den Lösungen wie auch an den neu entstandenen Problemen, sind die Dermatologen maßgeblich und die Psychologen folgerichtig beteiligt.

Die Kulturanthropologen mischen sich aktiv ein. Sie liefern den kulturellen Überbau. Umberto Ecco monografiert 2004 [12] über die Schönheit und 2007 über die Hässlichkeit [13]. Und die Debatte über das Alter bricht los. Das klassische Altertum bietet zwei Modelle, dasjenige der Verwerfung durch Aristoteles und das der Wertschätzung von Cicero. Entscheidend scheint aber immer der erste Aspekt, und den bietet die Haut. So schildert es die Monografie „Das Alter“ von Pat Thane 2005 [14]. Aging und Anti-Aging sind also primär dermatologischer Art und die Machbarkeit des Dekors hat ihr neues Tummelfeld.

Ja, Anti-Aging von der einen Seite konvergiert gleichsam mit dem Jugendwahn von anderseits. Niemand scheint ausgeschlossen und die Gegenwartsschrunpfung erlebt eine weitere hohe Zeit.

\section{Abstract}

\section{Cultural History and Dermatology}

Skin and Dermatology take their origin in the ancient times when the myths were formed. This may be demonstrated by the myth of the flied Satyr Marsyas. The bare cadaver acts as a metaphor for domination, suppression and for the loss of its person and individuality. The separated skin stands for fertility and rhythmic renewal according of the symbol of the snake sloughing its skin. These metaphors are still active up to the present in psychodermatology, in the new philosophy of the skin and in the upcoming debate on beauty, ugliness and aging.

\section{Literatur}

1 Publius Ovidius Naso. Metamorphosen. UB Nr. 356. Stuttgart: Reclam, 1971

2 Anzieu D. Das Haut-Ich. Frankfurt a. M.: Suhrkamp, 1991

3 Dawkins R. The Selfish Gene. Oxford: University Press, 1976

4 Assmann J, Hölscher T. Kultur und Gedächtnis. Frankfurt a. M.: Suhrkamp, 1988

5 Gschnait F, Exel W. Haut und Seele. Wien: Ueberreuter, 2002

6 Schipperges $H$. Kleine Kulturgeschichte der Haut. Rupperto Carola 1968; 20: 3-10

7 Jung EG. Kleine Kulturgeschichte der Haut. Darmstadt: Steinkopff, 2007

8 Jung EG. Haut und Kultur, auch ein Gang des Geistes. Akt Dermatol 2008; 34: 437-441

9 Condrau G, Schipperges H. Unsere Haut. Zürich: Kreuz-Verlag, 1994

10 Benthien C. Haut, Literaturgeschiche, Körperbilder, Grenzdiskurse. Reinbek: Rowohlt, 1999

11 Mittag H. Die Haut im medizinischen und kulturgeschichtlichen Kontext. Marburg: Völker \& Ritter, 2001

12 Eco U. Die Geschichte der Schönheit. München: Hanser, 2004

13 Eco U. Die Geschichte der Hässlichkeit. München: Hanser, 2007

14 Thane P. Das Alter. Eine Kulturgeschichte. Darmstadt: Primus, 2005 